\title{
Influence of wavy surfaces on coherent structures in a turbulent flow
}

\section{Conference Paper}

\section{Author(s):}

Kuhn, Simon; Wagner, Carsten; Rudolf von Rohr, Philipp

Publication date:

2007

Permanent link:

https://doi.org/10.3929/ethz-b-000003136

Rights / license:

In Copyright - Non-Commercial Use Permitted

Originally published in:

Experiments in Fluids 43(2-3), https://doi.org/10.1007/s00348-007-0262-x 


\title{
Influence of wavy surfaces on coherent structures in a turbulent flow
}

\author{
Simon Kuhn · Carsten Wagner • \\ Philipp Rudolf von Rohr
}

Received: 18 July 2006/ Revised: 8 January 2007 / Accepted: 8 January 2007 / Published online: 3 February 2007

(C) Springer-Verlag 2007

\begin{abstract}
We describe how outer flow turbulence phenomena depend on the interaction with the wall. We investigate coherent structures in turbulent flows over different wavy surfaces and specify the influence of the different surface geometries on the coherent structures. The most important contribution to the turbulent momentum transport is attributed to these structures, therefore this flow configuration is of large engineering interest. In order to achieve a homogeneous and inhomogeneous reference flow situation two different types of surface geometries are considered: (1) three sinusoidal bottom wall profiles with different amplitude-to-wavelength ratios of $\alpha=2 a / \Lambda=0.2$ $(\Lambda=30 \mathrm{~mm}), \alpha=0.2(\Lambda=15 \mathrm{~mm})$, and $\alpha=0.1$ $(\Lambda=30 \mathrm{~mm})$; and (2) a profile consisting of two superimposed sinusoidal waves with $\alpha=0.1 \quad(\Lambda$ $=30 \mathrm{~mm}$ ). Measurements are carried out in a wide water channel facility (aspect ratio 12:1). Digital particle image velocimetry (PIV) is performed to examine the spatial variation of the streamwise, spanwise and wall-normal velocity components in three measurement planes. Measurements are performed at a Reynolds number of 11,200 , defined with the half channel height $h$ and the bulk velocity $U_{\mathrm{B}}$. We apply the method of snapshots and perform a proper orthogonal decomposition (POD) of the streamwise, spanwise, and wall-normal velocity components to extract the most dominant flow structures. The structure of the most dominant eigenmode is related to counterrotating, streamwise-oriented vortices. A qualitative
\end{abstract}

S. Kuhn · C. Wagner · P. Rudolf von Rohr $(\bowtie)$

Institute of Process Engineering, ETH Zurich,

Sonneggstrasse 3, 8092 Zurich, Switzerland

e-mail: vonrohr@ipe.mavt.ethz.ch comparison of the eigenfunctions for different sinusoidal wall profiles shows similar structures and comparable characteristic spanwise scales $\Lambda_{z}=1.5 \mathrm{H}$ in the spanwise direction for each mode. The scale is observed to be slightly smaller for $\alpha=0.2(\Lambda=15 \mathrm{~mm})$ and slightly larger for $\alpha=0.2(\Lambda=30 \mathrm{~mm})$. This scaling for the flow over the basic wave geometries indicates that the size of the largest structures is neither directly linked to the solid wave amplitude, nor to the wavelength. The characteristic spanwise scale of the dominant eigenmode for the developed flow over the surface consisting of two superimposed waves reduces to $0.85 \mathrm{H}$. However, a scale in the order of $1.3 \mathrm{H}$ is identified for the second mode. The eigenvalue spectra for the superimposed waves is much broader, more modes contribute to the energy-containing range. The turbulent flow with increased complexity of the bottom surface is characterized by an increased number of dominant large-scale structures with different spanwise scales.

\section{Introduction}

Technical and geophysical relevant flows are characterized by high Reynolds numbers and complex boundaries. The turbulent flow over these rough or structured surfaces is associated with increased transport of species (heat or mass) and momentum. Differently shaped wavy walls as boundaries of the flow resemble the wall complexity in a well-defined manner and therefore serve as test cases for the study of wall influence on turbulence. We investigate the 
turbulent flow between a flat top and a complex bottom wall and describe the influence of different surface geometries on large-scale coherent structures in the region of the flow above the wall shear layer. In order to achieve a homogeneous and inhomogeneous reference flow situation two different types of surface geometries are considered: (1) three sinusoidal bottom wall profiles with different amplitude-to-wavelength ratios; and (2) a profile consisting of two superimposed sinusoidal waves.

The flow over a train of solid waves is connected to a developing shear layer, formed by the separation of the flow shortly after the wave crest, which extends over the whole wavelength (Cherukat et al. 1998). For smooth walls flow-oriented vortical eddies have been associated with large Reynolds stresses and with the production of turbulence in the viscous region close to the wall (Brooke and Hanratty 1993). Günther and Rudolf von Rohr (2003), Kruse et al. (2003), and Kruse et al. (2006) investigated the structure and dynamics of turbulent motions in the outer part of the wall shear layer in a developed turbulent flow over waves and identified flow-oriented large-scale structures.

The literature on the stability of a sheared flow over solid waves suggests the Görtler mechanism (Görtler 1940; Saric 1994) or the Craik-Leibovich type-2 mechanism (Phillips and Wu 1994; Phillips et al. 1996) to produce or catalyse spanwise-periodic longitudinal vortices. The Görtler mechanism is based on a boundary-layer instability induced by the local wall curvature which then forms steady, flow-oriented, counter-rotating vortices (Görtler vortices). In the works of Phillips and Wu (1994) and Phillips et al. (1996) longitudinal flow structures are explained by a Craik-Leibovich type-2 (CL-2) instability. This instability is caused by the periodicity of the bounding surface rather than its local curvature. The CL-2 instability is referred to as catalysing longitudinal flow structures.

We address the characteristic scales and eigenvalue spectra of large-scale structures in the turbulent flow over differently shaped complex surfaces by means of a POD of the velocity field obtained by stereoscopic PIV. Thus we describe the influence of different surface geometries on these large-scale coherent structures.

\section{Flow description}

We consider the turbulent flow between a solid complex bottom surface and a flat top wall. The investigated bottom surfaces are three different sinusoidal walls with amplitude-to-wavelength ratios of $\alpha=2 a$ l
$\Lambda=0.1(\Lambda=30 \mathrm{~mm}), \alpha=0.2(\Lambda=30 \mathrm{~mm})$, and $\alpha=0.2$ $(\Lambda=15 \mathrm{~mm})$, respectively a surface consisting of two superimposed sinusoidal waves with $\alpha=0.1$ $(\Lambda=30 \mathrm{~mm})$. The two types of complex surfaces and the used coordinate system are depicted in Fig. 1. The streamwise coordinate direction is denoted by $x$, the vertical direction by $y$, and $z$ is the spanwise direction. The corresponding velocity components are denoted as $u, v$, and $w$. The profile of the wavy walls can be calculated by

$y_{w}(x)=a \sin \left(\frac{2 \pi x}{\Lambda}\right)$,

the superposition of the two sinusoidal waves is given by

$y_{w}(x, z)=a \sin \left(\frac{2 \pi x}{\Lambda}\right) \sin \left(\frac{2 \pi z}{\Lambda}\right)$.

The flow over the wavy walls is homogeneous in spanwise direction, whereas for the flow over the superimposed waves inhomogeneous flow conditions are achieved. The turbulent flow is characterized by the Reynolds number

$R e_{h}=\frac{U_{\mathrm{B}} h}{v}$

where $v$ denotes the kinematic viscosity, $h$ is the half height of the channel, and the bulk velocity $U_{\mathrm{B}}$ is given by

$U_{\mathrm{B}}=\frac{1}{2 h-y_{w}} \int_{y_{w}}^{2 h} U\left(x_{\xi}, y\right) \mathrm{d} y$

where $x_{\xi}$ denotes an arbitrary $x$-location and $y_{w}$ describes the profile of the complex surface.

\section{Experiments}

The measurements are carried out in the channel facility depicted in Fig. 2. The working fluid is deionized and filtered water, the channel facility is made of anodized aluminium, PVC, and Schott BK-7 glass. All parts are positioned in a welded stainless steel frame. The flow loop and frequency controlled pumps are designed for turbulence measurements with light sheet techniques at Reynolds numbers up to 21,000 , defined with the bulk velocity $U_{\mathrm{B}}$ and the half channel height $h$. The measurements presented in this paper are performed at a Reynolds number of 11,200, earlier studies 


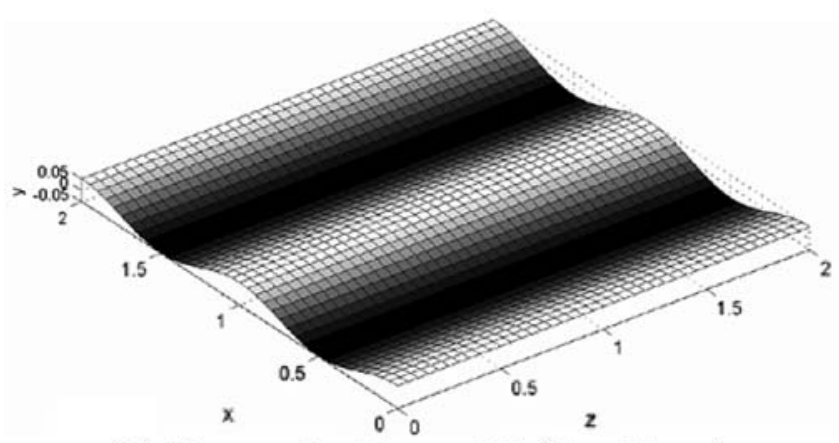

(a) Wavy wall with $\alpha=0.1(\Lambda=30 \mathrm{~mm})$

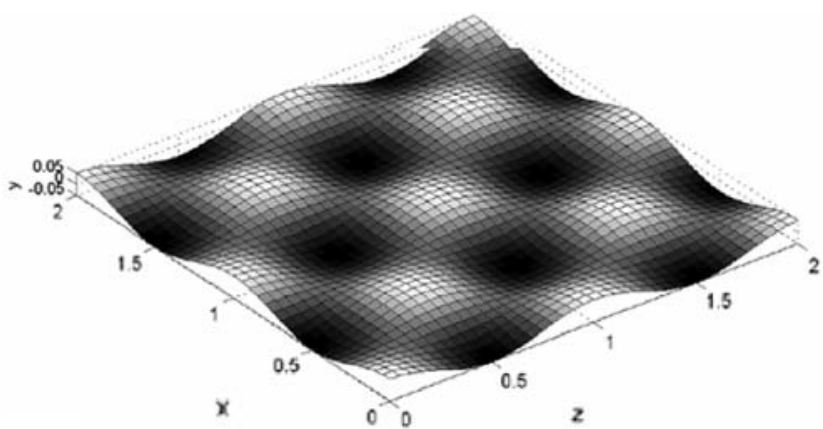

(b) Superimposed waves with $\alpha=0.1(\Lambda=30 \mathrm{~mm})$

Fig. 1 Profiles of the complex surfaces

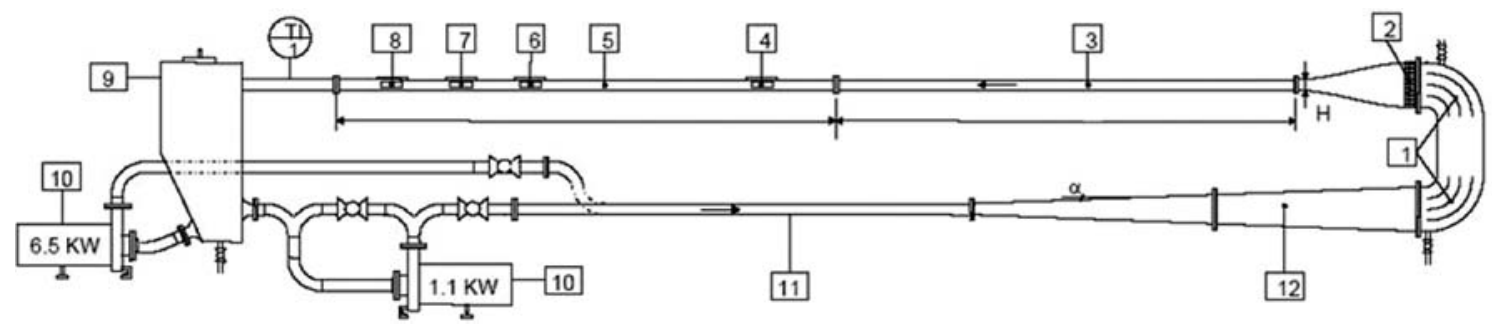

Fig. 2 Channel flow facility consisting of 1 turning elbows; 2 honeycomb; 3 flat-walled entrance section; 5 test section with complex bottom and flat top wall; 4, 6-8 optical viewports; 9 reservoir; 10 frequency controlled pumps; 11 pipe and 12 diffusor

showed no Reynolds number dependency for flow conditions at Reynolds numbers above 8,000 (Kruse 2005). The full height of the channel, $H$, is $30 \mathrm{~mm}$, and its aspect ratio (width $B$ to height $H$ ) is $12: 1$. The bottom wall in the test section can be interchanged between the four complex surfaces. Gauge measurements confirm that the milled PVC walls deviate less than $0.33 \%$ from the ideal sinusoidal shape. Optical access is provided at four streamwise positions through viewing ports, positioned at both sidewalls and at the flat top wall. Measurements are performed for a hydrodynamically developed turbulent flow after the 50th wave crest $(\Lambda=30 \mathrm{~mm})$, respectively after the 100th wave crest $(\Lambda=15 \mathrm{~mm})$. Digital PIV is performed to examine the spatial variation of the streamwise, spanwise and wall-normal velocity components (Adrian 1991; Raffel et al. 1998; Westerweel 1997; Westerweel and van Oord 2000). Measurements are performed in the $(x, y)-,(x, z)-$, and $(y, z)$-plane at a Reynolds number of 11,200 . For the $2 \mathrm{D} / 2 \mathrm{C}$ measurements the flow is seeded with hollow glass spheres with a mean diameter of $10 \mu \mathrm{m}$ (density: $1.03 \mathrm{~g} / \mathrm{cm}^{3}$ ), for the 2D/3C measurements $50 \mu \mathrm{m}$ polyamide particles (density: $1.01 \mathrm{~g} / \mathrm{cm}^{3}$ ) are used for seeding. We consider an ensemble of 1,000 consecutive images pairs in all measurement planes acquired at a frame rate of $4 \mathrm{~Hz}$. A flashlamp-pumped dual Nd:YAG-laser provides the pulse light source, a 10-bit CCD camera with a pixel- resolution of $1,280 \times 1,024$ pixels $^{2}$ is used for the velocity measurements. The measurement system consisting of the laser, the laser optics, and the camera, is positioned on a traverse that allows vertical adjustment with an accuracy of approximately $10 \mu \mathrm{m}$. The measurement accuracy of PIV is influenced by the particle image diameter, the particle image density, the particle image displacement and the velocity gradient within the interrogation area. Thus the total error can be subdivided into errors resulting from the image processing (interrogation scheme) and errors resulting from the image recording. Due to the considered interrogation algorithm the error level for the image processing is estimated to be below $10^{-2}$ pixels (Scarano and Riethmuller 2000; Scarano 2002). The error caused by the optical imaging is determined to be $0.01 \%$ (Kruse 2005). Thus the uncertainty of the PIV measurements resulting from the experimental setup, image acquisition and image processing is estimated to be in the order of $1 \%$.

\section{Results}

\subsection{POD analysis}

We use the method of snapshots (Sirovich 1987) and perform a proper orthogonal decomposition (POD) 
or Karhunen-Loève (KL) transformation of the measured velocity field (Berkooz et al. 1993). Reynolds averaging is applied and the velocity field $\mathbf{U}=[u, v, w]^{\mathrm{T}}$ measured in the $\left(x_{i}, x_{j}\right)$-plane is decomposed into a mean and fluctuating part:

$\mathbf{U}\left(x_{i}, x_{j}, t\right)=\overline{\mathbf{U}}\left(x_{i}, x_{j}\right)+\mathbf{U}^{\prime}\left(x_{i}, x_{j}, t\right)$.

We consider discrete times $t_{i}$ with $i=1, \ldots, M$, and $1, \ldots, N$ discrete locations in the $\left(x_{i}, x_{j}\right)$-plane, where $N=m n$ with $x_{i}:(1, m)$ and $x_{j}:(1, n)$. The resulting set of spatiotemporal data can be written as the $(3 N \times M)$ matrix

$$
\mathbf{M}=\left[\begin{array}{cccc}
u_{11}^{\prime} & u_{12}^{\prime} & \ldots & u_{1 M}^{\prime} \\
u_{21}^{\prime} & u_{22}^{\prime} & \ldots & u_{2 M}^{\prime} \\
\ldots & \ldots & \ldots & \ldots \\
u_{N 1}^{\prime} & u_{N 2}^{\prime} & \ldots & u_{N M}^{\prime} \\
v_{11}^{\prime} & v_{12}^{\prime} & \ldots & v_{1 M}^{\prime} \\
v_{21}^{\prime} & v_{22}^{\prime} & \ldots & v_{2 M}^{\prime} \\
\ldots & \ldots & \ldots & \ldots \\
v_{N 1}^{\prime} & v_{N 2}^{\prime} & \ldots & v_{N M}^{\prime} \\
w_{11}^{\prime} & w_{12}^{\prime} & \ldots & w_{1 M}^{\prime} \\
w_{21}^{\prime} & w_{22}^{\prime} & \ldots & w_{2 M}^{\prime} \\
\ldots & \ldots & \ldots & \ldots \\
w_{N 1}^{\prime} & w_{N 2}^{\prime} & \ldots & w_{N M}^{\prime}
\end{array}\right]
$$

Using the method of snapshots the $(M \times M)$ covariance matrix reads

$\mathbf{C}_{i j}=\frac{1}{M}\left\langle\mathbf{M}_{i} \mathbf{M}_{j}\right\rangle, \quad i, j=1, \ldots, M$

where $\langle\cdot, \cdot\rangle$ denotes the Euclidian inner product. Since this covariance matrix is symmetric its eigenvalues, $\lambda_{i}$ $(i=1, \ldots, M)$, are non-negative, and its eigenvectors, $\varphi_{i}$ $(i=1, \ldots, M)$, form a complete orthogonal set. The orthogonal eigenfunctions are

$\Pi^{[k]}=\sum_{i=1}^{M} \phi_{i}^{[k]} \mathbf{M}_{i}, \quad k=1, \ldots, M$

where $\varphi_{i}^{[k]}$ is the $i$ th component of the $k$ th eigenvector. The total energy $E$ of the flow is obtained by the summation over the eigenvalues $\lambda_{i}(i=1, \ldots, M)$

$E=\sum_{i=1}^{M} \lambda_{i}$

The fractional contribution of each eigenfunction to the total energy is given by the fractional contribution of its associated eigenvalue

$\frac{E_{k}}{E}=\frac{\lambda_{k}}{\sum_{i=1}^{M} \lambda_{i}}$

Using only the first $\mathrm{K}$ most energetic eigenfunctions an approximation of the original data is given by
$\mathbf{U}_{j}^{\prime}=\overline{\mathbf{U}}+\sum_{i=1}^{K} a_{i} \Pi^{[i]}$,

where the coefficients $a_{\mathrm{i}}$ are calculated from the projection of the sample vector $\mathbf{U}_{j}^{\prime}$ onto the eigenfunction $\Pi^{[i]}$ :

$a_{i}=\frac{\mathbf{U}_{j}^{\prime} \cdot \Pi^{[i]}}{\Pi^{[i]} \cdot \Pi^{[i]}}$.

This operation corresponds to low-pass filtering and can be used to study the structure of the large-scale structures.

\subsection{Results in the $(x, y)$-plane}

The measurements in the $(x, y)$-plane are performed in a field of view which covers the whole region between the complex surface and the flat top wall, extending at least over one wavelength in streamwise direction [FOV $1.1 H$ (streamwise) $\times 1.0 H$ (vertical)]. The spatial resolution of the PIV data in this plane of measurement is $0.014 H$ (streamwise) $\times 0.015 H$ (vertical). Thus it is possible to determine the location of the most dominant flow structures in the flow field. Figure 3 depicts the contour plot of the streamwise component of the first eigenfunction obtained by a POD of $u / U_{\mathrm{B}}(x, y, z=0.5 B, t)$ for all wavy profiles at a Reynolds number of $R e_{h}=11,200$. The most energetic flow structure is located in the vicinity of the complex surface. The maxima is found at a location of $y / H=0.26$ for the profiles $\alpha=0.2(\Lambda=15 \mathrm{~mm})$ and $\alpha=0.1(\Lambda=30 \mathrm{~mm})$, for $\alpha=0.2(\Lambda=30 \mathrm{~mm})$ slightly above and for the superimposed waves slightly below that value. We identify contributions to the kinetic energy of $34 \%$ for the profile $\alpha=0.1(\Lambda=30 \mathrm{~mm})$, which decrease with decreasing wavelength to $24.6 \%$ for the profile $\alpha=0.2(\Lambda=15 \mathrm{~mm})$, respectively with increasing amplitude to $29.4 \%$ for the profile $\alpha=0.2$ $(\Lambda=30 \mathrm{~mm})$. The lowest value of $10.3 \%$ is found for the profile consisting of the superimposed waves. Figure 4 depicts the fractional and cumulative kinetic energy contribution of the streamwise eigenvalues. For the flow over the basic wavy profiles more than $50 \%$ of the total energy is captured by less than ten modes. With increasing wall complexity the eigenvalue spectrum becomes broader and therefore the number of important modes in the flow increases.

\subsection{Results in the $(x, z)$-plane}

The measurements in the $(x, z)$-plane are performed in a field of view which extends over a length corre- 
Fig. 3 Comparison of the first eigenfunction for a decomposition of $u$ / $U_{\mathrm{B}}(x, y, z=0.5 B, t)$ for a $\alpha=0.2(\Lambda=15 \mathrm{~mm})$, b $\alpha=0.2(\Lambda=30 \mathrm{~mm})$, c $\alpha=0.1(\Lambda=30 \mathrm{~mm})$, and d superimposed waves, $R e_{h}=11,200$

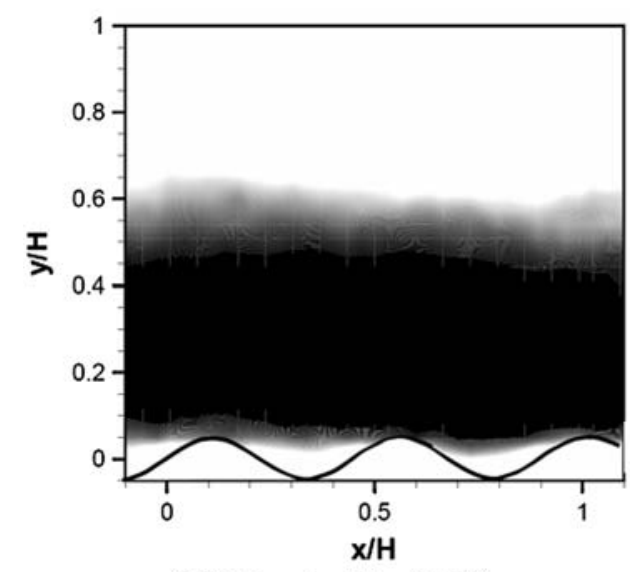

(a) $\Pi_{1, u} ; \lambda_{1} / E=24.6 \%$

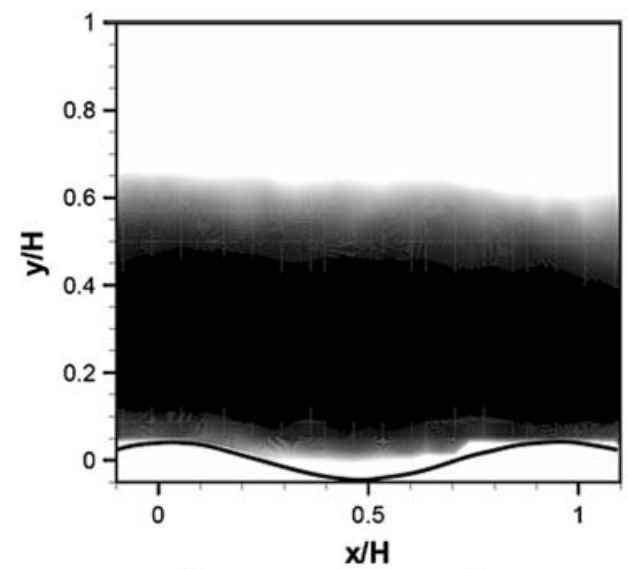

(c) $\Pi_{1, u} ; \lambda_{1} / E=34.0 \%$

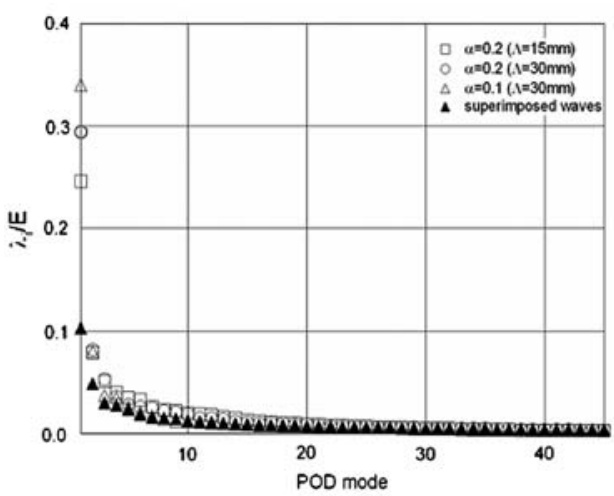

(a) $\lambda_{i, u}$

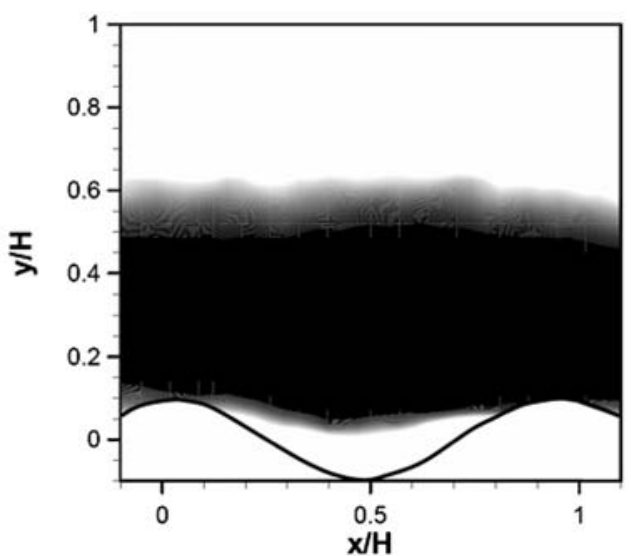

(b) $\Pi_{1, u} ; \lambda_{1} / E=29.4 \%$

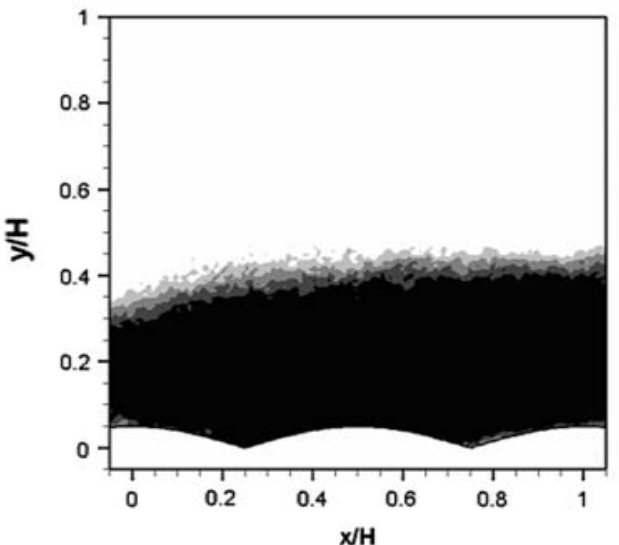

(d) $\Pi_{1, u} ; \lambda_{1} / E=10.3 \%$

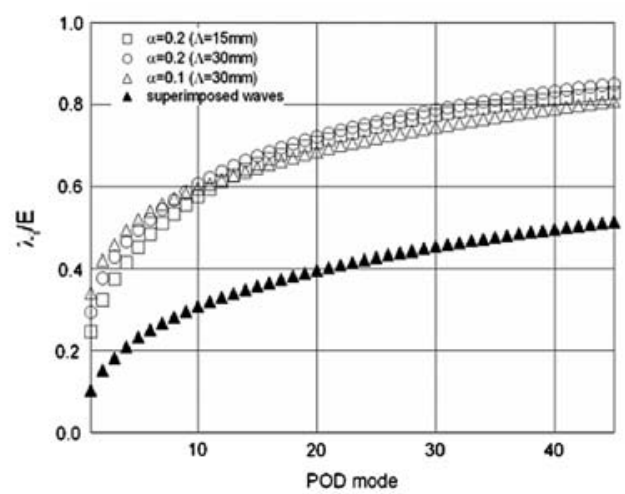

(b) $\lambda_{i, u}$
Fig. 4 a Fractional and b cumulative kinetic energy contribution from streamwise eigenvalues for a decomposition of $u / U_{\mathrm{B}}(x, y, z=0.5 B, t)$, $R e_{h}=11,200$ 
the basic wave profiles. A qualitative comparison of the eigenfunctions for the three sinusoidal profiles exhibits similar structures and a characteristic spanwise scale in the order of $\Lambda_{z}=1.5 \mathrm{H}$. This spanwise scale is also confirmed by the second eigenfunction. The highest energy contribution is found for the first mode of the profile $\alpha=0.1(\Lambda=30 \mathrm{~mm})$. Similar to the results in the $(x, y)$-plane the energy contribution reduces with decreasing wavelength $[\alpha=0.2(\Lambda=15 \mathrm{~mm})]$, respectively increasing amplitude $[\alpha=0.2(\Lambda=30 \mathrm{~mm})]$. For the profile of the superimposed waves the energy contribution of the first mode is $10.4 \%$. This value is larger than the one for the surfaces with an amplitudeto-wavelength ratio of $\alpha=0.2$, but smaller than for the profile $\alpha=0.1(\Lambda=30 \mathrm{~mm})$. The spanwise scale of the first eigenfunction for the superimposed waves is in the order of $0.85 \mathrm{H}$. However, this scaling is not confirmed by the second mode, which exhibits a larger scale in the order of $1.3 \mathrm{H}$, comparable to the spanwise distance of the first and second eigenmodes of the basic wave profiles. This supports the notion that by increasing the surface complexity the energy spectrum increases and thus the number of important modes exhibiting different scales is enlarged.

This is confirmed in Fig. 6 where the fractional and cumulative kinetic energy contribution of the streamwise eigenvalues for the decomposition $u / U_{\mathrm{B}}(x, y /$ $H=0.26, z, t)$ is depicted. The eigenvalue spectra of the basic sinusoidal profiles becomes broader with decreasing wavelength $[\alpha=0.2(\Lambda=15 \mathrm{~mm})]$, respectively increasing amplitude $[\alpha=0.2(\Lambda=30 \mathrm{~mm})]$. For the surface described by the superposition of two sinusoidal waves the broadest spectrum is found, although not so pronounced as the spectra in the $(x, y)$-plane (Fig. 4).

To quantitatively address the spanwise scaling of the large-scale structures we perform a streamwise averaging of the first two eigenfunctions as depicted in Fig. 7. To compare the scaling between the different basic wave profiles the curves are shifted in the homogeneous spanwise direction, so that one maximum coincides. We detect a characteristic spanwise scale of the first eigenfunction of $\Lambda_{z}=1.5 \mathrm{H}$ for the profile $\alpha=0.1(\Lambda=30 \mathrm{~mm})$. For the profile $\alpha=0.2$ $(\Lambda=30 \mathrm{~mm})$ with doubled amplitude the scaling increases to $\Lambda_{z}=1.7 \mathrm{H}$. A spanwise distance of $\Lambda_{z}$ $=1.3 \mathrm{H}$ is found for the profile $\alpha=0.2(\Lambda=15 \mathrm{~mm})$ with same amplitude but half the wavelength compared to $\alpha=0.1(\Lambda=30 \mathrm{~mm})$. These scalings are also confirmed by the second eigenmode depicted in Fig. 7c. Thus we identify a characteristic spanwise scale of the first two eigenfunctions which is almost independent of the wall geometry. The scaling of the large-scale structures depends neither solely on the amplitude nor the wavelength of the sinusoidal surface.

For the surface consisting of two superimposed sinusoidal waves the spanwise scale of the first eigenmode of $\Lambda_{z}=0.85 \mathrm{H}$ is confirmed by the averaging process. But for the second eigenfunction a larger spanwise distance of $\Lambda_{z}=1.3 \mathrm{H}$ is found. This scaling is comparable to the one of the basic sinusoidal profiles found for the first two dominant modes.

\subsection{Results in the $(y, z)$-plane}

The measurements in the $(y, z)$-plane are performed in a field of view which covers the whole region between the complex surface and the flat top wall and extends at least over a length corresponding to one channel height in spanwise direction. For the basic wave profiles the field of view is $1.0 H$ (vertical) $\times 1.6 H$ (spanwise) with a resulting spatial resolution of $0.017 \mathrm{H}$ (vertical) $\times 0.02 H$ (spanwise), for the superimposed waves a smaller field of view of $1.0 \mathrm{H}$ (vertical) $\times 1.0 \mathrm{H}$ (spanwise) with a resulting higher resolution of $0.012 H$ (vertical) $\times 0.017 H$ (spanwise) is chosen. The POD is applied to the velocity vector field $\mathbf{U} / U_{\mathrm{B}}(x)$ $\Lambda=0.5, y, z, t)$ to obtain structural information about the large-scale structures.

Figure 8 depicts the first eigenfunction for the profile $\alpha=0.2(\Lambda=15 \mathrm{~mm})$, exemplarily chosen for the basic wave profiles, and for the surface defined by the superposition of two sinusoidal profiles. The structure of the most dominant mode is that of counter-rotating vortices. Between these vortices fluid is transported to and away from the surface. The centers of the vortices are found in the lower half of the channel at a wallnormal distance which corresponds to the maxima of the first eigenfunctions of the decomposition in the $(x, y)$-plane (Fig. 3). The radius of the vortical fluid motion is slightly larger for the basic wave profiles, where a value of $0.8 \mathrm{H}$ is found. For the superimposed waves the radius decreases to $0.4 \mathrm{H}$. The spanwise scale of identical fluid movement (i.e. to or away from the surface) is equal to the spanwise scale of the dominant eigenmode found in the $(x, z)$-plane. Therefore we identify the large-scale structures present in the vicinity of the complex surface as streamwise-oriented, counter-rotating vortices exhibiting a characteristic spanwise scale.

For all surfaces we found that eigenfunctions corresponding to higher POD modes and thus lesser energy contribution show characteristic smaller spanwise scales that may likely be connected to the local wall curvature, i.e. the Görtler instabilities (Görtler 1940; Saric 1994). 
Fig. 5 Comparison of the first two eigenfunctions for a decomposition of $u / U_{\mathrm{B}}(x, y /$ $H=0.26, z, t)$ for $\alpha=0.2$

$(\Lambda=15 \mathrm{~mm})$ (first row), $\alpha=0.2(\Lambda=30 \mathrm{~mm})$ (second row $), \alpha=0.1(\Lambda=30 \mathrm{~mm})$ (third row), and for the superimposed waves (fourth row), $\operatorname{Re}_{h}=11,200$

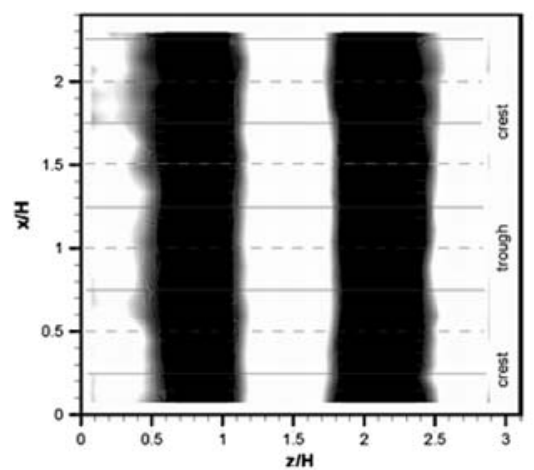

(a) $\Pi_{1, u} ; \lambda_{1} / E=8.9 \%$

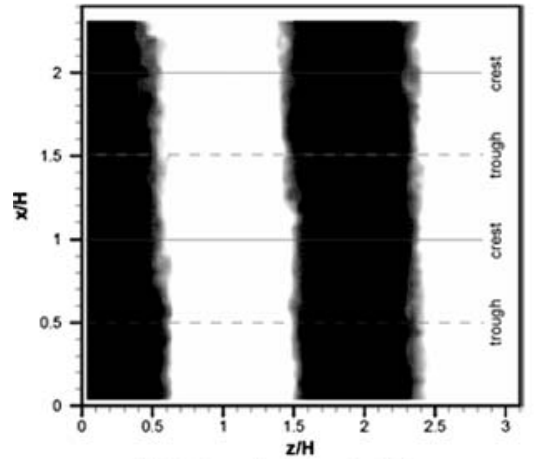

(c) $\Pi_{1, u} ; \lambda_{1} / E=8.8 \%$

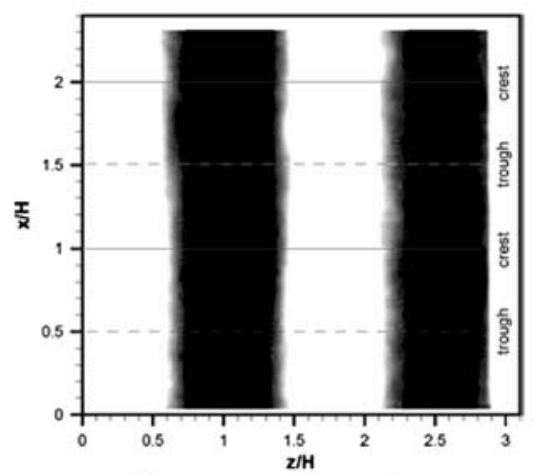

(e) $\Pi_{1, u} ; \lambda_{1} / E=14.3 \%$

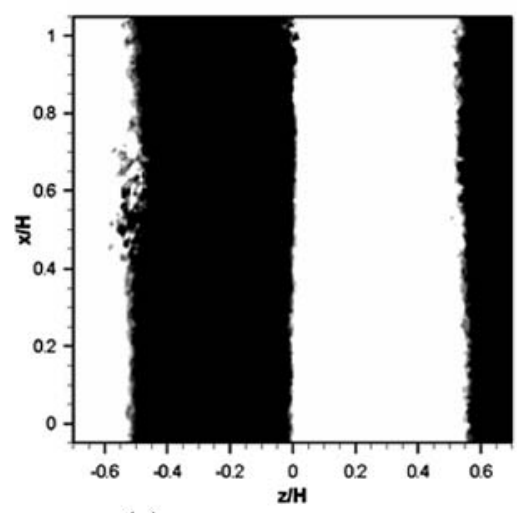

(g) $\Pi_{1, u} ; \lambda_{1} / E=10.4 \%$

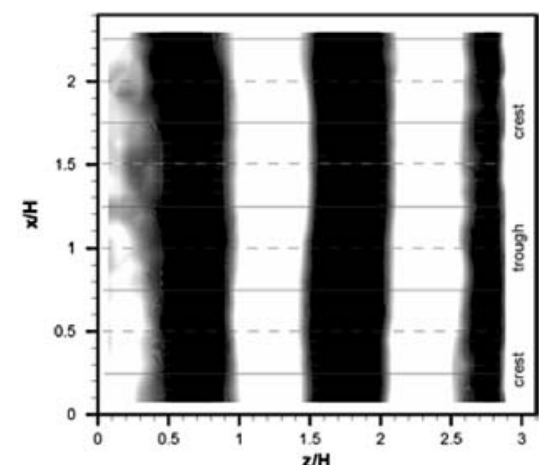

(b) $\Pi_{2, u} ; \lambda_{2} / E=7.5 \%$

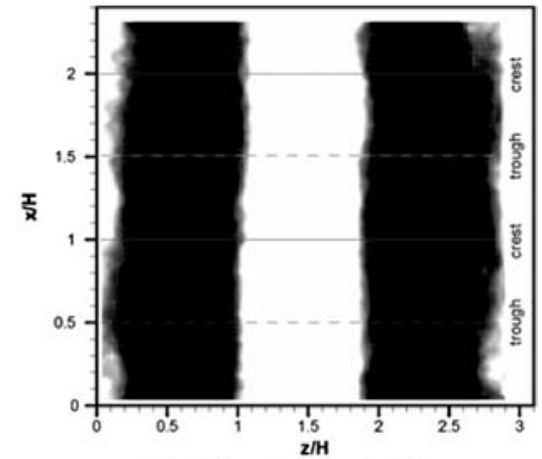

(d) $\Pi_{2, u} ; \lambda_{2} / E=8.4 \%$

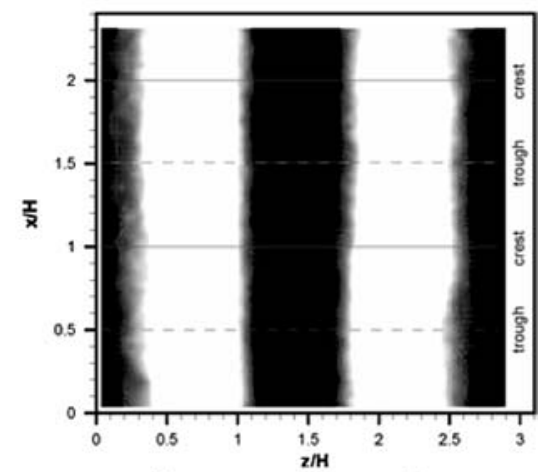

(f) $\Pi_{2, u} ; \lambda_{2} / E=13.0 \%$

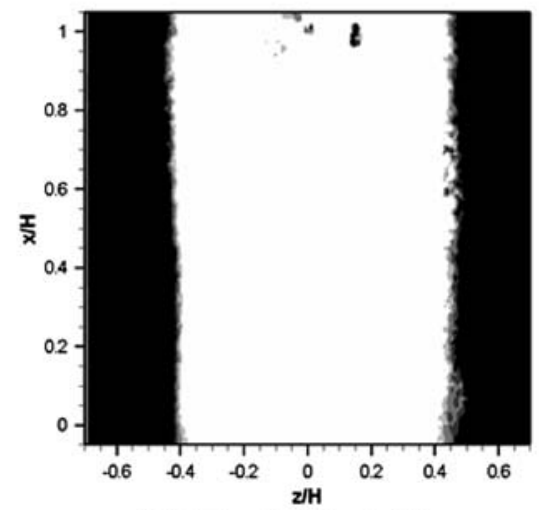

(h) $\Pi_{2, u} ; \lambda_{2} / E=6.9 \%$ 
Fig. 6 a Fractional and b cumulative kinetic energy contribution from streamwise eigenvalues for a decomposition of $u / U_{\mathrm{B}}(x, y /$ $H=0.26, z, t), R e_{h}=11,200$
Fig. 7 Comparison of streamwise-averaged eigenfunctions $\left\langle\Pi_{1, u}\right\rangle_{x}$ and $\left\langle\Pi_{2, u}\right\rangle_{x}$ for a decomposition of $u / U_{\mathrm{B}}(x, y / H=0.26, z, t)$ for $\mathbf{a}$ and $\mathbf{c}$ the wavy wall profiles and $\mathbf{b}$ and $\mathbf{d}$ the superimposed waves, $R e_{h}=11,200$

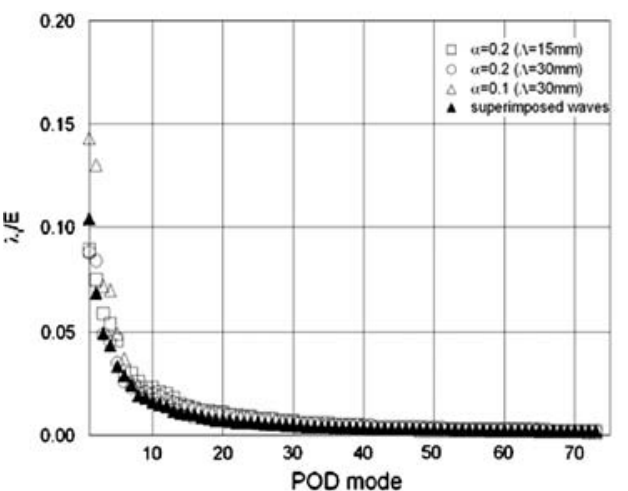

(a) $\lambda_{i, u}$

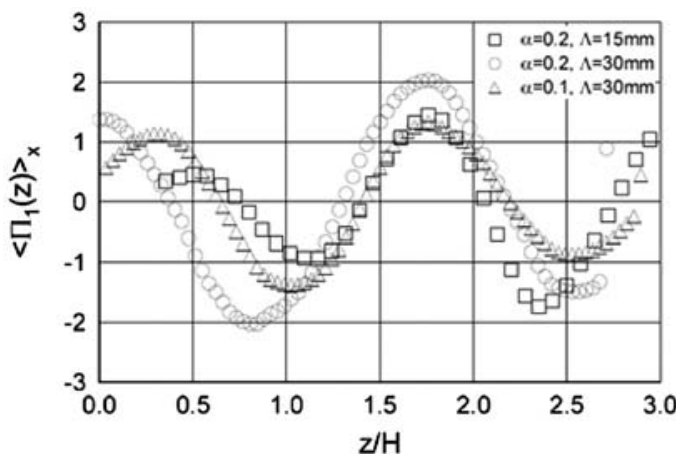

(a) $\left\langle\Pi_{1, u}\right\rangle_{x}$

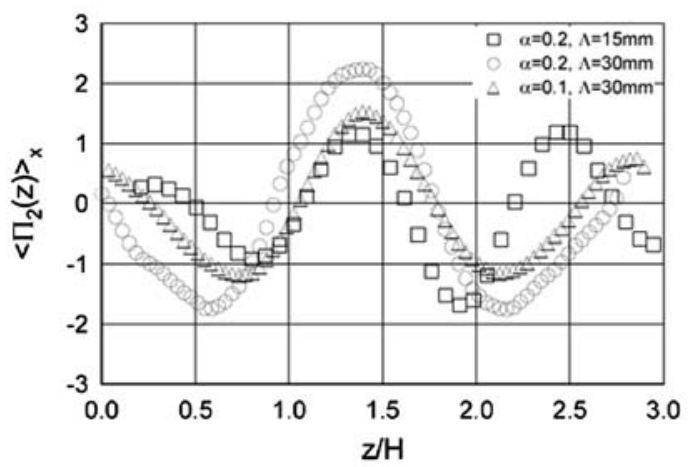

(c) $\left\langle\Pi_{2, u}\right\rangle_{x}$

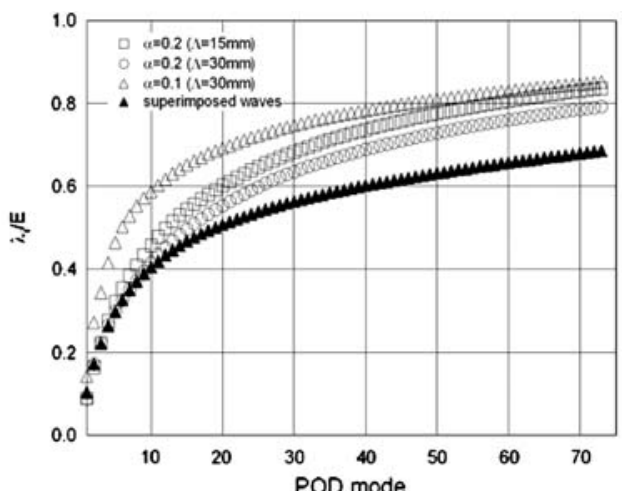

(b) $\lambda_{i, u}$

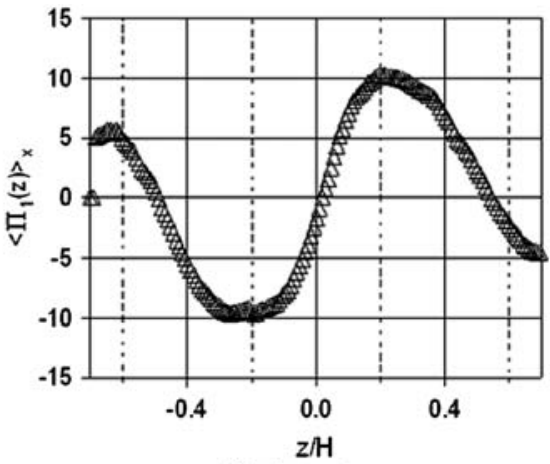

(b) $\left\langle\Pi_{1, u}\right\rangle_{x}$

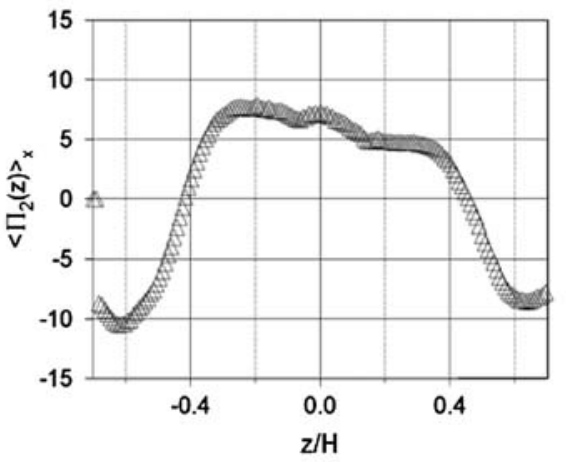

(d) $\left\langle\Pi_{2, u}\right\rangle_{x}$

\section{Conclusions}

In this study we addressed the influence of different surface geometries on the coherent structures in a turbulent flow. We obtained large image sequences in three planes of measurement of a turbulent channel flow at a Reynolds number of $R e_{h}=11,200$. We applied the method of snapshots and performed a POD of the velocity field to extract structural information of the coherent structures.

From this orthogonal decomposition of velocity fields we find similar large-scale structures in the vicinity of the complex surface. These large-scale structures are identified as streamwise-oriented, counter-rotating vortices exhibiting a characteristic scale in the spanwise coordinate direction. We quantitatively describe this spanwise scale by applying the POD onto the streamwise velocity component in the $(x, z)$-plane. We found similar spanwise scales in the order of $\Lambda_{z}=1.5 H$ for the basic wavy surfaces of different amplitude-to-wavelength ratios in the first two eigenmodes. In addition, the oscillation and the location of the first two streamwise-averaged eigenfunction extrema are nearly identical. This scaling indicates that the size of the largest structures neither depends solely on the solid wave amplitude, nor on the wavelength. 
Fig. 8 Comparison of the first eigenfunction for a decomposition of $\mathbf{U} / U_{\mathrm{B}}(x /$ $\Lambda=0.5, y, z, t)$ for $\mathbf{a} \alpha=0.2$ $(\Lambda=15 \mathrm{~mm})$ and $\mathbf{b}$ the superimposed waves, $R e_{h}=11,200$

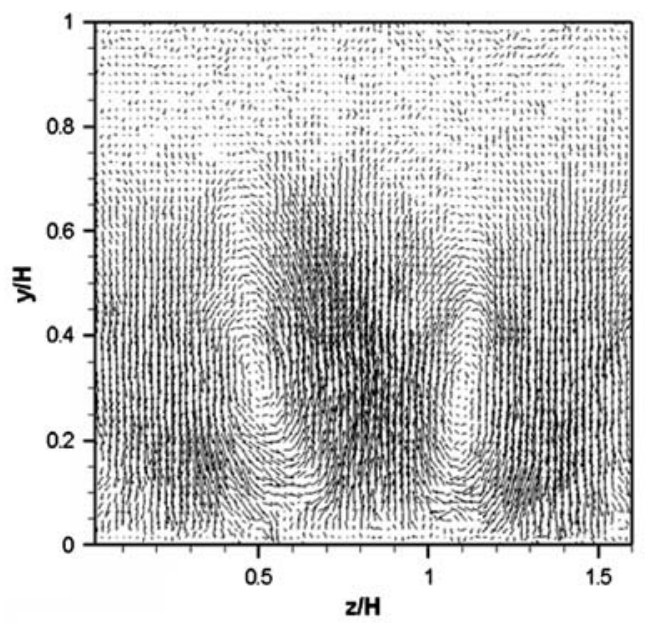

(a) $\Pi_{1,(v, w)} ; \lambda_{1} / E=3.5 \%$

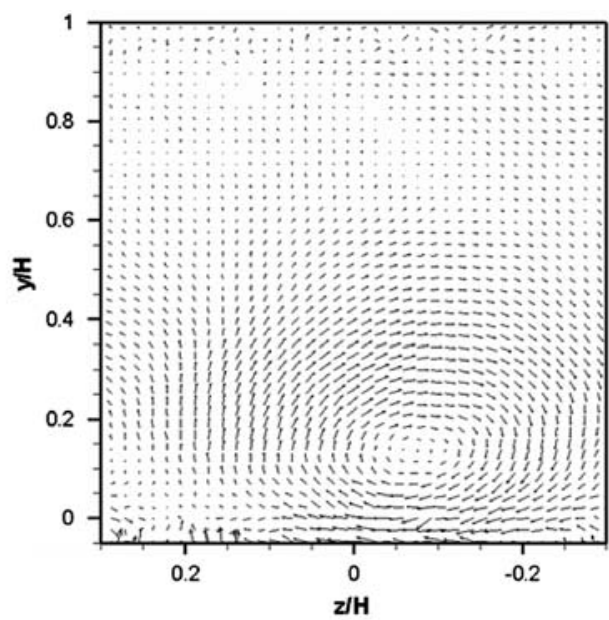

(b) $\Pi_{1,(v, w)} ; \lambda_{1} / E=3.8 \%$
For the profile described by the superposition of two sinusoidal waves a spanwise scale of $\Lambda_{z}=0.85 H$ was identified for the first eigenmode. However, this scaling is not confirmed by the second eigenfunction where a spanwise distance of $\Lambda_{z}=1.3 \mathrm{H}$ is observed. Additionally, the location of the extrema of the second eigenmode is shifted by a spanwise distance of $z$ l $H=0.4$.

The eigenvalue spectra from the orthogonal decomposition in the $(x, y)$ - and $(x, z)$-plane become increasingly broader for the profile with doubled amplitude $[\alpha=0.2(\Lambda=30 \mathrm{~mm})]$, half the wavelength $[\alpha=0.2(\Lambda=15 \mathrm{~mm})]$, and the superimposed waves. Thus by increasing the surface complexity more modes contribute to the energy containing range.

We conclude that by increasing the complexity of the bottom surface and thus altering the flow homogeneity from homogeneous in spanwise coordinate direction as for the basic wave profiles to completely inhomogeneous as for the superimposed waves the energy spectrum of the turbulent flow and the number of significant modes is increased. The flow over the superimposed waves can be described as the superposition of dominant eigenmodes with different spanwise scales.

Acknowledgment We gratefully acknowledge financial support from the Swiss National Science Foundation (SNF). Measurement technology is partially provided by ILA GmbH.

\section{References}

Adrian RJ (1991) Particle-imaging techniques for experimental fluid mechanics. Annu Rev Fluid Mech 23:261-304

Berkooz G, Holmes P, Lumley JL (1993) The proper orthogonal decomposition in the analysis of turbulent flows. Annu Rev Fluid Mech 25:539-575
Brooke JW, Hanratty TJ (1993) Origin of turbulence-producing eddies in a channel flow. Phys Fluids A5(4):1011-1022

Cherukat P, Na Y, Hanratty TJ, McLaughlin JB (1998) Direct numerical simulation of a fully developed turbulent flow over a wavy wall. Theoret Comp Fluid Dynam 11:109-134

Görtler H (1940) Über eine dreidimensionale Instabilität laminarer Grenzschichten an konkaven Wänden. Nachr Ges Wiss Göttingen, Math-Phys Klasse Neue Folge I 2:1-26

Günther A, Rudolf von Rohr P (2003) Large-scale structures in a developed flow over a wavy wall. J Fluid Mech 478:257-285

Kruse N (2005) Isothermal and non-isothermal turbulent flow over solid waves. PhD thesis, ETH Zurich, Switzerland, Url http://www.e-collection.ethbib.ethz.ch/show?type=diss\&nr= 16031

Kruse N, Günther A, Rudolf von Rohr P (2003) Dynamics of large-scale structures in turbulent flow over a wavy wall. J Fluid Mech 485:87-96

Kruse N, Kuhn S, Rudolf von Rohr P (2006) Wavy wall effects on turbulence production and large-scale modes. J Turbulence 7(31):1-24

Phillips WRC, Wu Z (1994) On the instability of wave-catalysed longitudinal vortices in strong shear. J Fluid Mech 272:235254

Phillips WRC, Wu Z, Lumley JL (1996) On the formation of longitudinal vortices in a turbulent boundary layer over wavy terrain. J Fluid Mech 326:321-341

Raffel M, Willert C, Kompenhans J (1998) Particle image velocimetry. A practical guide. Springer, Heidelberg

Saric WS (1994) Görtler vortices. Annu Rev Fluid Mech 26:379_ 409

Scarano F (2002) Iterative image deformation methods in PIV. Meas Sci Technol 13:R1-R19

Scarano F, Riethmuller ML (2000) Advances in iterative multigrid PIV image processing. Exp Fluids 29:S51-S60

Sirovich L (1987) Turbulence and the dynamics of coherent structures. Part I. Coherent structures. Q Appl Math XLV:561-571

Westerweel J (1997) Fundamentals of digital particle image velocimetry. Meas Sci Technol 8:1379-1392

Westerweel J, van Oord J (2000) Stereoscopic PIV measurements in a turbulent boundary layer. In: Stanislas M, Kompenhans J, Westerweel J (eds) Particle image velocimetry, progress toward industrial application. Kluwer, Dordrecht 